\title{
An Artificial Neural Network Model for Classification of Epileptic Seizures Using Huang- Hilbert Transform
}

\author{
Shaik.Jakeer Husain ${ }^{1}$ and Dr.K.S.Rao ${ }^{2}$ \\ ${ }^{1}$ Dept. of Electronics and Communication Engineering, Vidya Jyothi Institute of \\ Technology, Hyderabad India
}

\begin{abstract}
Epilepsy is one of the most common neurological disorders characterized by transient and unexpected electrical disturbance in the brain. In This paper the EEG signals are decomposed into a finite set of band limited signals termed as Intrinsic mode functions. The Hilbert transom is applied on these IMF's to calculate instantaneous frequencies. The 2nd,3rd and 4th IMF's are used to extract features of epileptic signal. A neural network using back propagation algorithm is implemented for classification of epilepsy.
\end{abstract}

An overall accuracy of $99.8 \%$ is achieved in classification..

\section{KEYWORDS}

Electroencephalogram(EEG),Hilbert-Huang transform(HHT), Instantaneous frequency (ifs), intrinsic mode function (IMF)

\section{INTRODUCTION}

Epilepsy is a neurological disorder with wide prevalence of about 1 to $2 \%$ of the world's population[1][2] .It is characterized by sudden recurrent and transient disturbances of perception or behavior resulting from excessive synchronization of cortical neuronal networks; it is a neurological condition in which an individual experiences chronic abnormal bursts of electrical discharges in the brain. The hallmark of epilepsy is recurrent seizures termed "epileptic seizures". Epileptic seizures are divided by their clinical manifestation into partial or focal, generalized, unilateral and unclassified seizures Focal epileptic seizures involve only part of cerebral hemisphere and produce symptoms in corresponding parts of the body or in some related mental functions. Generalized epileptic seizures involve the entire brain and produce bilateral motor symptoms usually with loss of consciousness. Both types of epileptic seizures can occur at all ages. Generalized epileptic seizures can be subdivided into absence (petit mal) and tonic-colonic (grand mal) seizures Monitoring brain activity through the electroencephalogram (EEG) has become an important tool in the diagnosis of epilepsy. The recorded EEG data are often distorted by other signals, called artifacts, whose origins are either of physiological or technical nature. There have been proposed many techniques to identify, separate, and suppressthese artifacts from the EEG signals. One of the most common techniques used for that purpose is Independent Component 
Analysis (ICA) However, ICA does not work well for highly non-stationary artifacts such as muscle artifacts. Several Time-frequency methods have been proposed for the detection and classification of seizures, most notably the Wavelet transform. These are non-stationary methods[4]. In parallel ,probabilistic methods such as various entropies, fractal dimensions, lyapunov exponents have been utilized. The above mentioned techniques are non linear[5].

In 1998, Norden E. Huang et al. proposed a new method for analyzing non-stationary data [3][6]. This technique is known as the Huang-Hilbert transform (HHT). The HHT uses a novel prepossessing algorithm, the Empirical Mode Decomposition (EMD), to decompose an arbitrary signal into a sum of so-called Intrinsic Mode Functions (IMF's) and a residue[8]. Based on the inherent characteristics of the IMF's ,the EMD can be used for denoising and detrending purposes. In order to denoise the signals, it has been suggested in to remove the IMF's that are considered to be dominated by noise[9][10]. In this paper we propose a new method which selects the IMF's which were based on the weighted mean Frequency(MF) of each IMF's. From the selected IMF's features are extracted, and these features are classified using Neural Networks.

\section{METHODOLOGY}

\subsection{Description of the EEG Database}

EEG data considered for this work is obtained from University of Bonn EEG database which is available in public domain containing three different cases: 1) healthy, 2) epileptic subjects during seizure-free interval (interictal), 3) epileptic subjects during seizure interval (ictal) [7]. Each set contains 100 single channel EEG segments of $23.6 \mathrm{sec}$ duration. Sampling frequency is $173.61 \mathrm{~Hz}$, so each segment contains $\mathrm{N}=4096$ samples. All these EEG segments are recorded with the same 128- channel system and that are digitized by 12 bit A/D convertor.

\subsection{Hilbert-Huang transform (HHT).}

The HHT consists of two parts: empirical mode decomposition (EMD) and Hilbert spectral analysis (HSA). This method is potentially viable for nonlinear and nonstationary data analysis, especially for time frequency energy representations. The physically meaningful way to describe such a system is in terms of the instantaneous frequency, which will reveal the intra-wave frequency modulations. The easiest way to compute the instantaneous frequency is by using the Hilbert transform. In a time time series, it is possible to define an analytic signal using hilbert transform which constitues the imaginary part Its amplitude and phase is time dependent. For this reason, three new concepts were introduced, the instantaneous amplitude and the phase functions. The time derivative of phase function is called instantaneous frequency function. Even with the Hilbert transform, defining the instantaneous frequency still involves considerable controversy. It would lead to the problem of having frequency values being all equally likely to be positive and negative for any given time series (positive or negative energy). As a result, the past applications of the Hilbert transform are all limited to the narrow band-passed signals, which have with the same number of extreme values and zero crossings. But filtering is a linear operation, altering its harmonics and creating a distortion of the waveform. To avoid this situation, before Hilbert transform it is necessary to use the so called empirical mode decomposition (EMD) introduced by N.Huang in 1998[3]. Table-1 provides the comparisons of transformation. 
This is an adaptive method, with posterior defined basis from the decomposition ,By definition, an Intrinsic Mode functions satisfies two conditions. These are: a) The number of extrema and the number of zero crossings may differ by no more than one b) The local average defined by the average of the maximum and minimum envelopes is zero.

TABLE 1:COMPARISON BETWEEN FOURIER, WAVELET AND HHT

$\begin{array}{llll} & \text { Fourier } & \text { Wavelet } & \text { HHT } \\ \text { Basis } & \text { A priori } & \text { A priori } & \text { adaptive } \\ \text { Frequency } & \begin{array}{l}\text { Convolution: } \\ \text { global, uncertainty }\end{array} & \begin{array}{l}\text { Convolution: } \\ \text { global, uncertainty }\end{array} & \begin{array}{l}\text { Differential: local } \\ \text { certainty }\end{array} \\ \text { Presentation } & \text { Energy-frequency } & \begin{array}{l}\text { Energy-time- } \\ \text { frequency }\end{array} & \begin{array}{l}\text { Energy-time- } \\ \text { frequency } \\ \text { Nonlinear }\end{array} \\ \text { Non stationa } & \text { No } & \text { No } & \text { Yes } \\ \text { Feature Extraction } & \text { No } & \text { Yes } & \text { Yes } \\ & & \begin{array}{l}\text { Discrete: no } \\ \text { Continuous } \\ \text { :yes }\end{array} & \text { Yes }\end{array}$

\section{Empirical Mode Decomposition (EMD)}

The basic idea of EMD is that each time series consists of different simple intrinsic modes of oscillations. Each intrinsic mode, linear or nonlinear, represents a simple oscillation, which will have the same number of extreme values and zero-crossings and the oscillation will also be symmetric with respect to the 'local mean'. These functions are the mono-components or the intrinsic mode functions (IMF). The process of acquiring the IMF's is called sifting and it's described below

1.Identify all the maxima and minima of $\mathrm{x}(\mathrm{t})$

2. Generate its upper and lower envelopes, $X_{\text {up }}(t)$ and

$\mathrm{X}_{\text {low }}(\mathrm{t})$ with cubic spline interpolation

3.Calculate the point-by-point mean from the upper and lower envelopes, by using $m(t)=\left(X_{\text {up }}(t)\right.$ $\left.+\mathrm{X}_{\text {low }}(\mathrm{t})\right) / 2$.

4. Extract the detail, $d(t)=x(t)-m(t)$

5. Test the following two conditions of $d(t): a)$ if $d(t)$ meets the two conditions related to the IMF definition (mentioned previously), an IMF is derived.

Replace $x(t)$ with the residual $r(t)=x(t)-d(t)$;b) if $d(t)$ is not an IMF, replace $x(t)$ withd $(t)$, and 6. Repeat steps 1 to 5 until a monotonic residual, or a single maximum or minimum residual satisfying fallowing stopping criterion.

$$
\text { Standard Deviation }(\mathrm{SD})=\sum_{t=0}^{T} \frac{\left|d_{k-1}(t)-d_{k}(t)\right|^{2}}{d_{k}^{2}(t)}
$$


At the end of this process, the signal $\mathrm{x}(\mathrm{t})$ can be expressed as follows:

$$
\mathrm{x}(\mathrm{t})=\sum_{j=1}^{N^{-}} c_{j}(t)+r_{N}(t)
$$

where $\mathrm{N}$ is the number of intrinsic modes, $\mathrm{r}$ ( $\mathrm{t}$ ) denote $\mathrm{s}$ the final residue, which can be interpreted as the DC component of the signal. $\mathrm{C} \mathrm{j}(\mathrm{t})$ are he intrinsic modes, orthogonal to each other and all have zero means. The decomposed signal shown in Fig 1

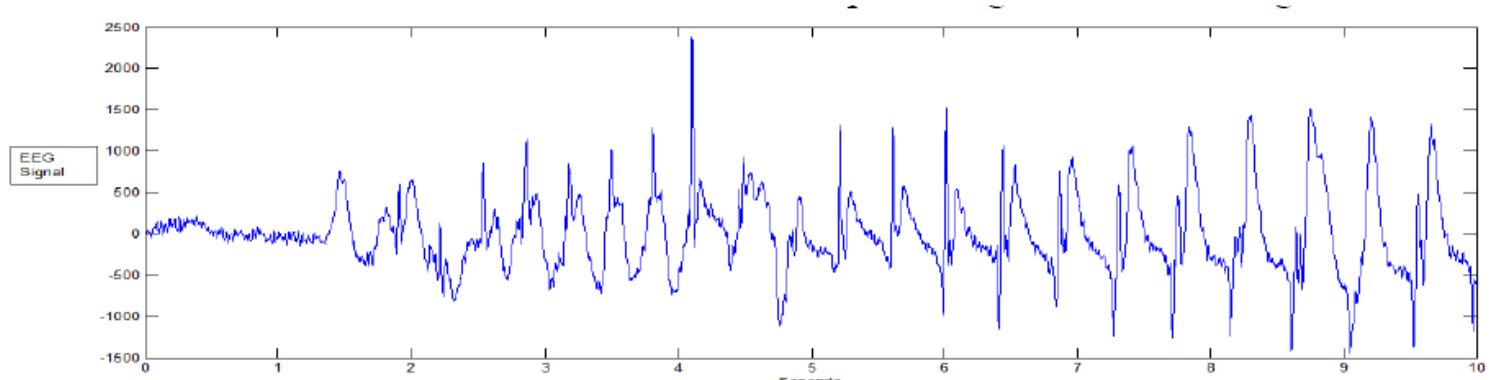

\section{FIG 1(A) EEG SIGNAL BEFORE DECOMPOSITION}
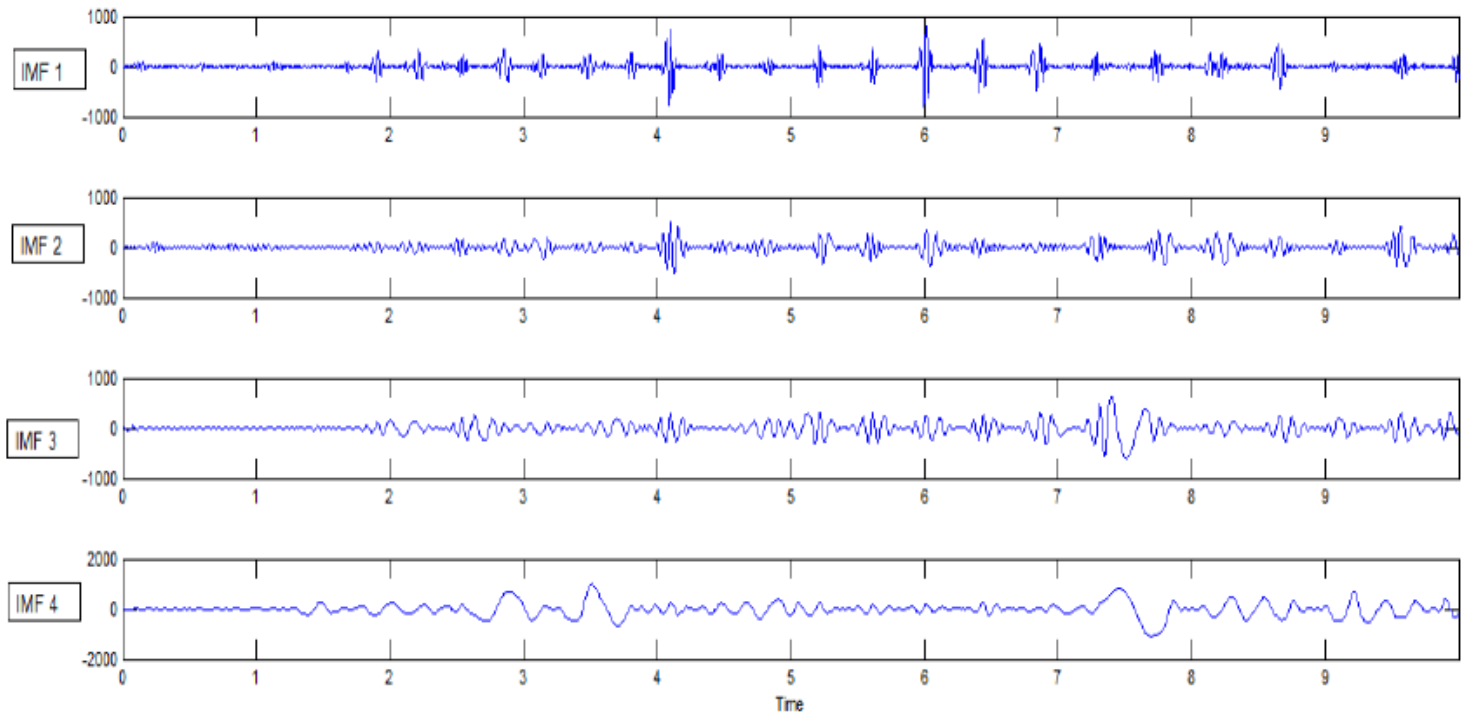

Fig 1(b)Decomposed EEG Signal(IMF1-IMF4) 
International Journal on Soft Computing (IJSC) Vol. 5, No. 3, November 2014
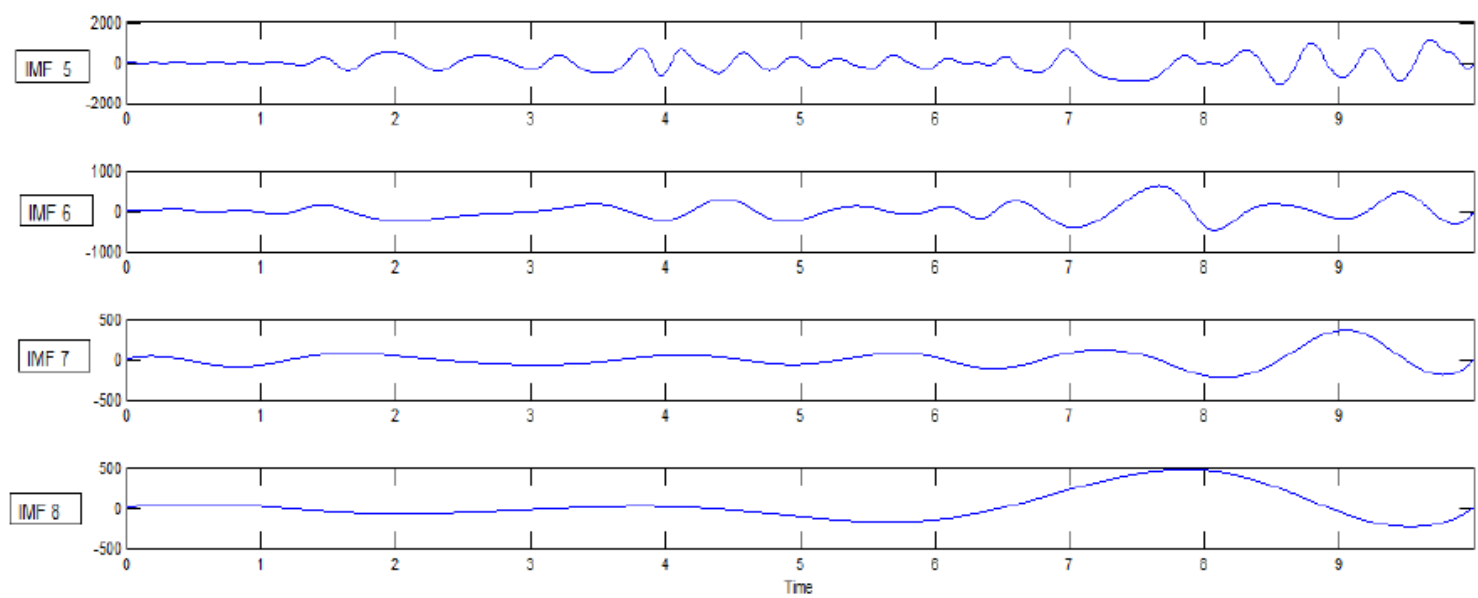

Fig.1(c).Decomposed EEG Signal(IMF7-IMF8)

Now, Hilbert Transform can be applied to every single intrinsic function.

\section{Hilbert Transform}

Given a real signal $\mathrm{x}(\mathrm{t})$,its complex representation is

$$
z(t)=x(t)+i x_{H}(t)
$$

where $\mathrm{XH}_{\mathrm{H}}(\mathrm{t})$ is the Hilbert transform of $\mathrm{x}(\mathrm{t})$, given by

$$
\mathrm{x}_{\mathrm{H}}(\mathrm{t})=\frac{1}{\pi} P \int_{-\infty}^{\infty} x \frac{(s)}{t-s} d s
$$

with $\mathrm{P}$ the Cauchy principal value of the integral. Equation (2) can be rewritten in an exponential form as

$$
Z(t)=A(t) \mathrm{e}^{i \Psi(t)}
$$

where $A(t)=\sqrt{x(t)^{2}+x_{H}(t)^{2}}$

$$
\Psi(t)=\arctan \left(\frac{x_{H}(t)}{x(t)}\right)
$$

The time derivative of (5) is

$$
\dot{Z}(t)=A(t) \mathrm{e}^{i \psi(t)}(i w t)+\mathrm{e}^{i \psi(t)} \dot{A}(t)
$$

where $\mathrm{w}(\mathrm{t})$ is the instantaneous angular frequency, which bydefinition is the time derivative of the instantaneous angle. 


$$
w(t)=\dot{\psi}(t)=\frac{d}{d t} \arctan \left(\frac{x_{H}(t)}{x(t)}\right)
$$

Hence the instantaneous frequency can be defined as

$$
f(t)=\frac{w(t)}{2 \pi}
$$

From Instantaneous frequencies computing weightedmean Frequency(MF)

$$
\mathrm{MF}=\frac{\sum_{i=1}^{L} A_{1}(i) f_{1}(i)^{2}}{\sum_{i=1}^{L} A_{1}(i) f_{1}(i)}
$$

A1- Instantaneous Amplitude, f1-Instantaneous Frequency

Following four features are extracted from each IMF

I. Rate of change of amplitude envelopes of IMF's

II. Weighted mean Frequency(MF)

III. Inter quartile range in each IMF

The IQR is defined by IQR $=\mathrm{Q} 3-\mathrm{Q} 1 \ldots \ldots$ (12)

Where, Q1 and Q3 are the first and third quartile respectively.

\section{Median absolute deviation in each IMF}

The median absolute deviation is the mean of the absolute deviations of a set of data about the data's mean. For a sample size $N$ and the mean distribution $\mathrm{x}$, the median absolute deviation is defined by(MAD)

$$
\frac{1}{N} \sum_{i=1}^{N}\left|x_{i}-\bar{x}\right|
$$

\section{EXPERIMENTAL RESULTS AND DISCUSSION}

Fig 2shows the extracted IMF's (left-side of the figure) and Instantaneous frequency of corresponding IMF' s (Right side of the Figure) of Seizure signal.Fig 3corresponds to Nonseizure data. 
International Journal on Soft Computing (IJSC) Vol. 5, No. 3, November 2014

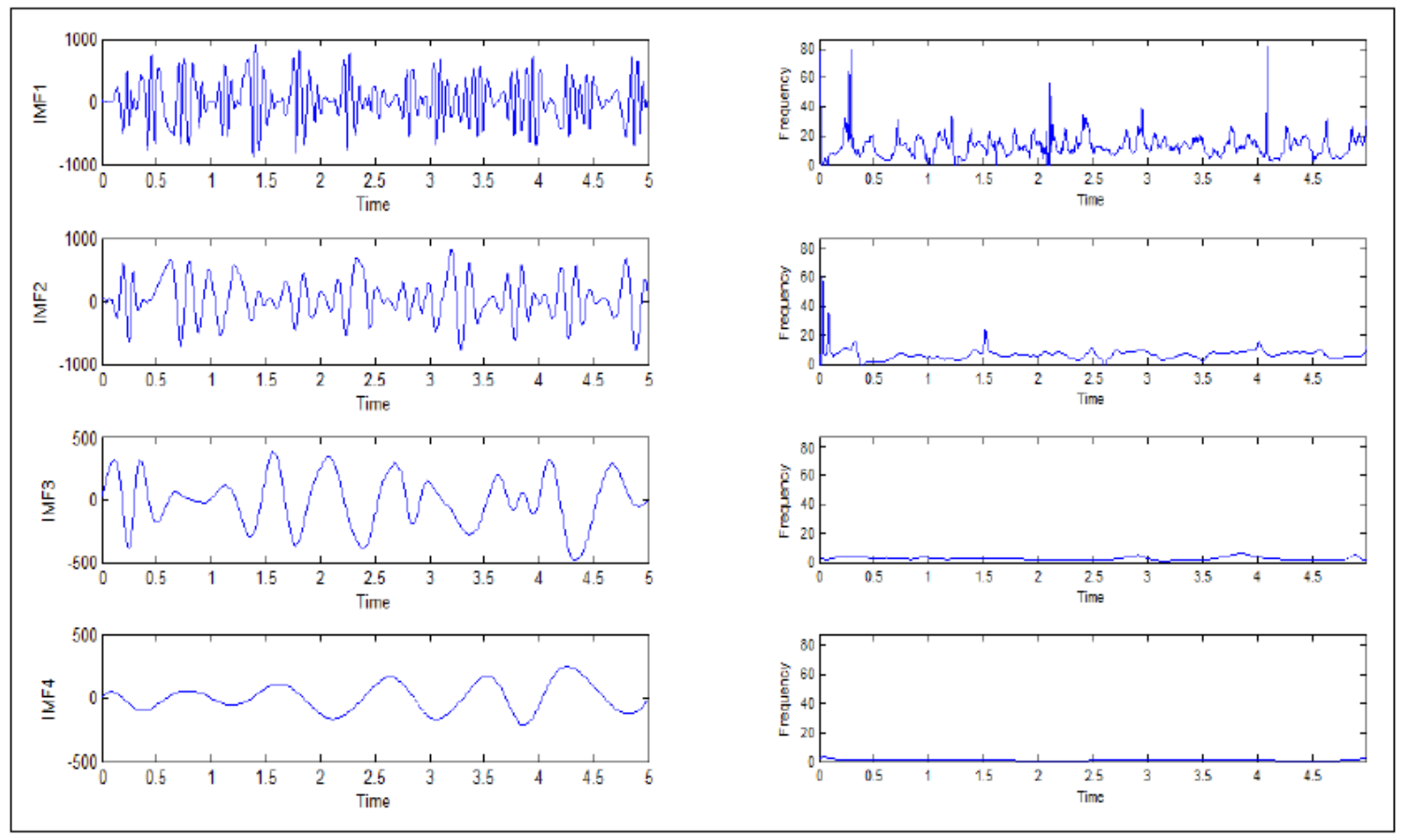

Fig.2.Instantaneous frequency (IF) functions of IMF's of: seizure EEG signal.

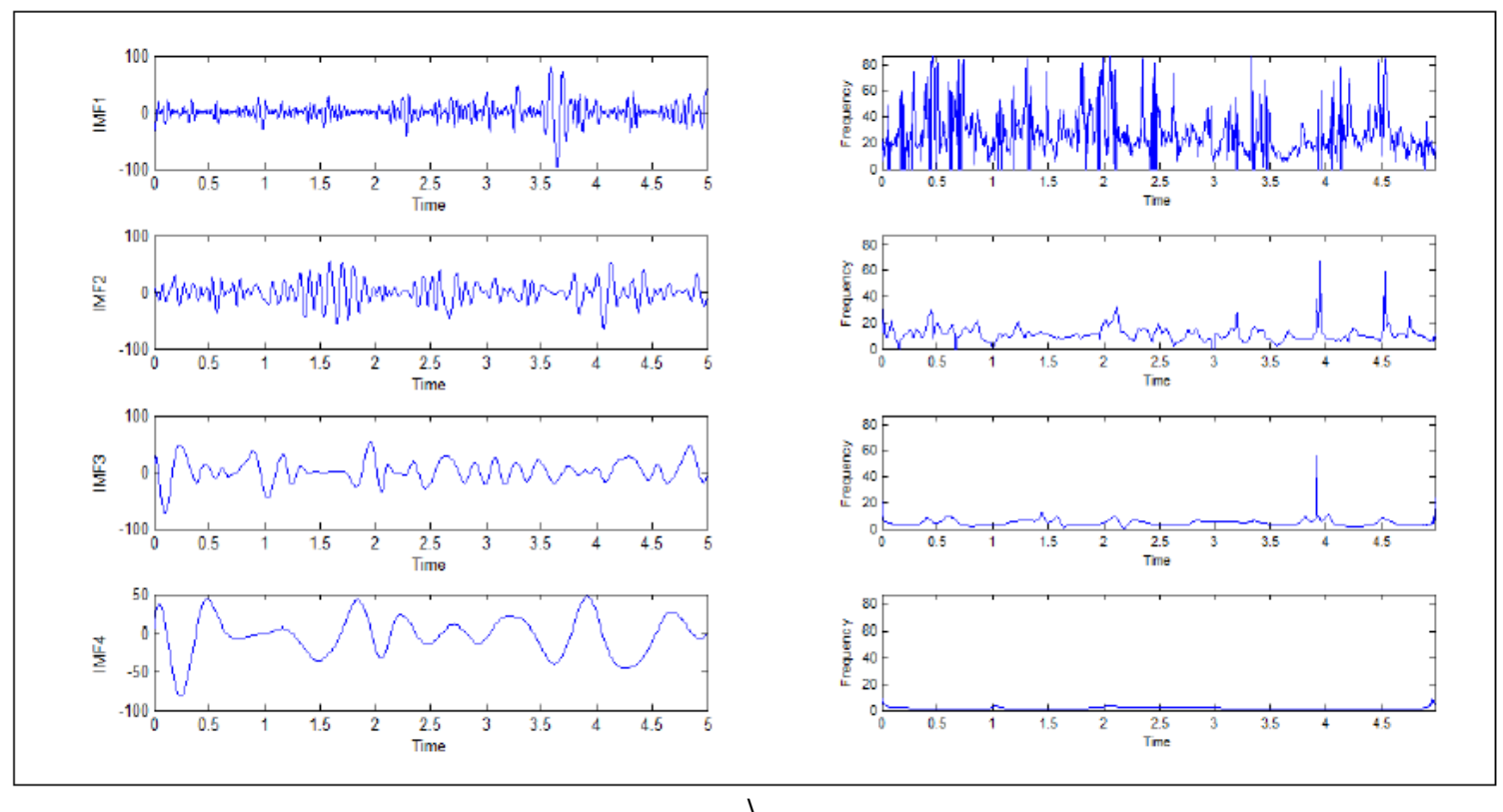

Fig. 3.Instantaneous frequency (IF) functions of IMF's of: non seizure EEG signal.

Table-2 enlists the Mean Instantaneous frequencies of seizure and Non-seizure data IMF's. 
Table -2 Mean Frequency of IMF's of seizure and non seizure EEG

\section{Intrinsic modes}

Weighted frequency Non seizure Data(Hz)

29.54

11.65

4.82

1.95

0.74

0.29

0.22

0.07

\section{Weighted frequency seizure Data(Hz)}

15.88

6.65

2.56

1.22

0.64

0.30

0.19

0.07

The mean weighted frequency suggest that the IMF's numbered one to four is useful to discriminate the seizure and non seizure data. IMF-1 is having high frequency oscillations and artifacts.We are considering IMF's numbered 2 to 4 for the following features;1.Mean weighted frequency;2. Rate of change of envelope amplitude;3 Inter quartile range ;4. Mean absolute deviation.These features are extracted from selected IMF's. From each epoch of $5 \mathrm{~s}$ duration;12 features extracted. Seizure data of 400 epochs and non seizure data of 400 epochs are considered. These features are applied to a Neural network with 12 input neurons, one output neuron and one hidden layer. We used the Feed Forward Back propagation algorithm.following.

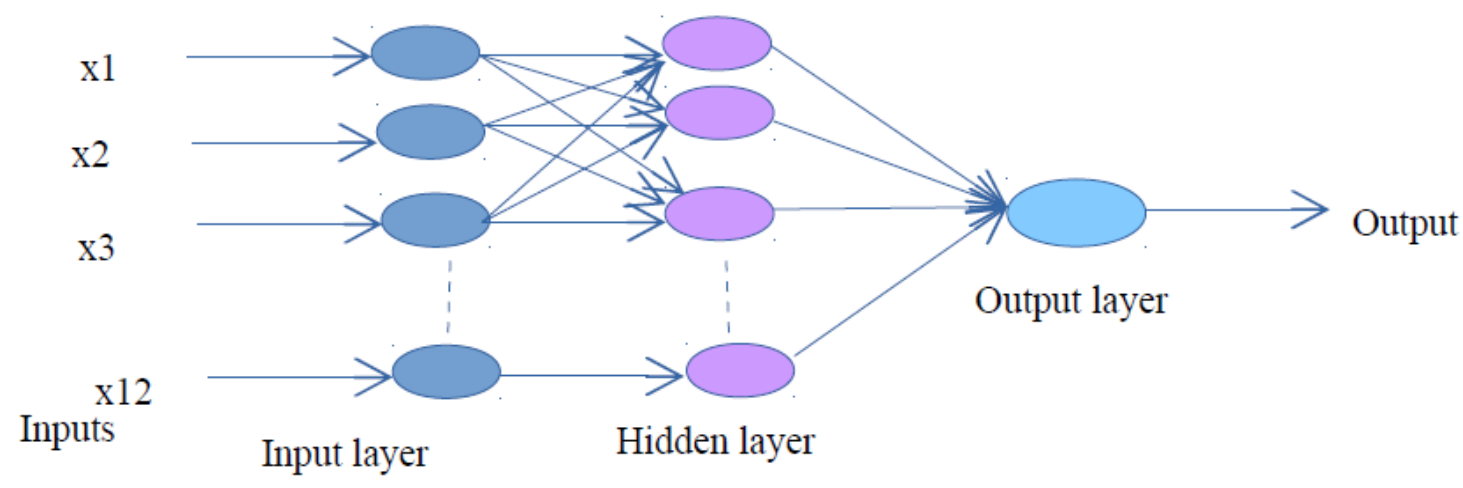

FIG. 4 NEURAL NETWORK ARCHITECTURE 


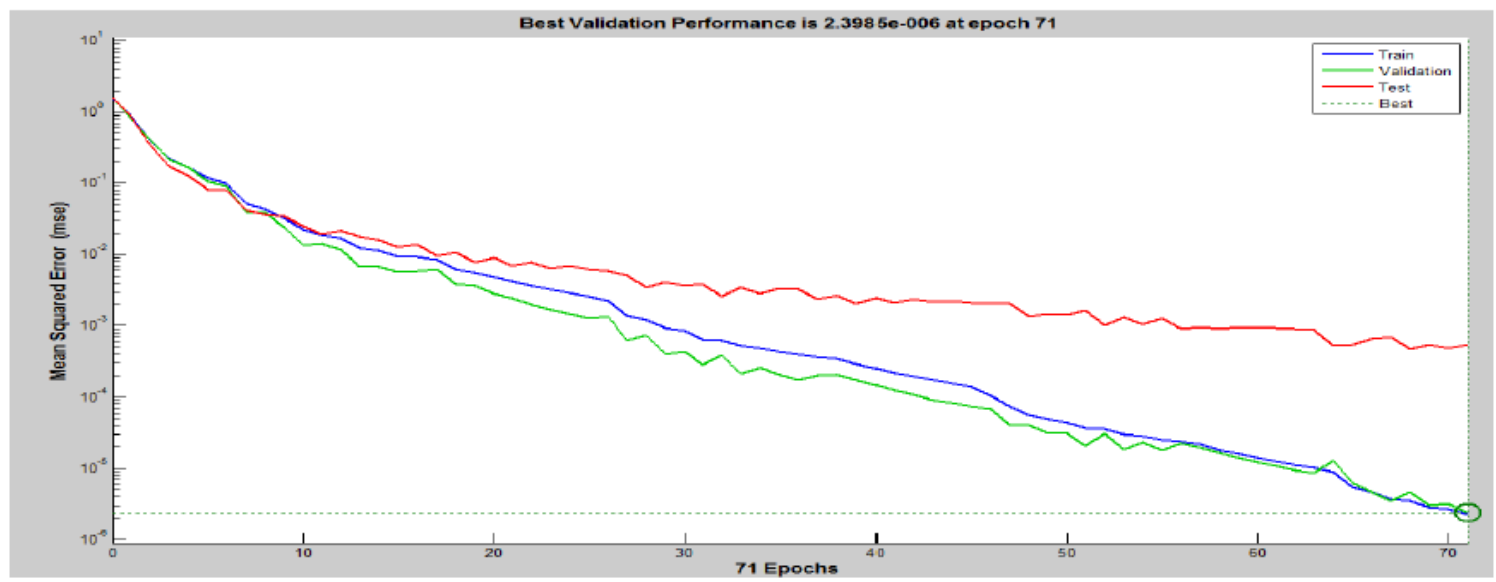

FIG. 5 TRAINING PERFORMANCE

Fig.4 show the neural network and Fig.5gives the training performance. We got the best validation performance as $2.3985 \mathrm{e}-006$ at epoch 71 .The trained network is simulated with seizure and non seizure data. We got one epoch as false negative and zero false positives. Figure 6 shows the simulation results of 400 epochs of seizure and 400 epochs of seizure-free data.

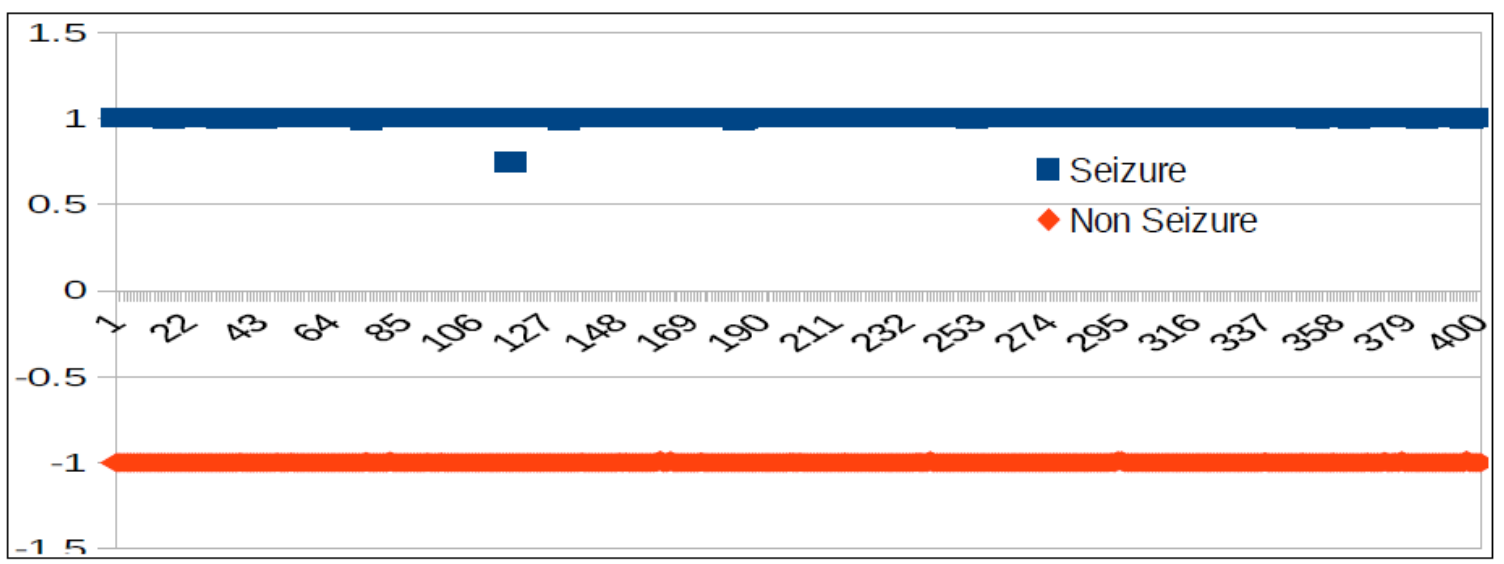

Fig. 6 Simulation Results of Neural Network with Non seizure (represented with -1) and seizure (represented with +1$)$.

Sensitivity(SE): number of true positive(TP) decisions / number of actually positive cases. Specificity (SP): number of true negative (TN)decisions / number of actually negative cases. Total classification accuracy(TCA): number of correct decisions / total number of cases.

TABLE-3 SENSITIVITY, SPECIFICITY AND CLASSIFICATION ACCURACY

$\begin{array}{lllllll}\text { TP } & \text { FN } & \text { SE } & \text { TN } & \text { FP } & \text { SP } & \text { TCA } \\ 399 & 01 & 99.75 & 400 & 0 & 100 \% & 99.8 \%\end{array}$


From TABLE 3, it is observed that sensitivity, specificity and accuracy of the proposed method is superior. The experimental results for both EEG data, therefore, confirm that the proposed method is well organized and attains a reasonable dominance with respect to classification accuracy. We believe that the proposed system can be very helpful to the physicians for their final decision on their patient's treatments. By using such an efficient tool, they can make very accurate decisions.

\section{CONCLUSION}

Seizure and non seizure EEG signals are analyzed in this paper. Empirical mode decomposition method used to Extract IMF's. Based on mean weighted Frequency, we selected IMF's for feature Extraction. The extracted features are classified using neural network. The proposed techniques in this paper are used for EEG signal processing to come up with a new original tool that gives physicians the possibility to diagnose brain functionality abnormalities. The proposed system bears the potential of providing several credible benefits such as fast diagnosis, high accuracy, good sensitivity and specificity.

To establish the clinical use for this seizure detection scheme it is necessary to test on out of sample data sets. Papers in this format must not exceed twenty (20) pages in length. Papers should be submitted to the secretary AIRCC. Papers for initial consideration may be submitted in either .doc or .pdf format. Final, camera-ready versions should take into account referees' suggested amendments.

\section{REFERENCES}

[1] J. Gotman., "Automatic recognition of epileptic seizures in the EEG," Clinical Neurophysiology, vol. 54, pp. 530-540, 1982

[2] J.Gotman., "Automatic seizure detection: improvements and evaluation," Clinical Neurophysiology, vol. 76, pp. 317-324, 1990

[3] N.E. Huang, Z. Shen, S.R. Long, M.L. Wu, H.H. Shih,Q. Zheng, N.C. Yen, C.C. Tung, and H.H. Liu, "TheEmpirical Mode Decomposition and Hilbert Spectrumfor Nonlinear and Nonstationary Time Series Analysis,” Proc. Roy. Soc., vol. 454, pp. 903 - 995, 1998.

[4] Y.U. Khan, J. Gotman, "Electroencephalogram Wavelet based automatic seizure detection iintracerebral", Clinical Neurophysiology, vol. 114, pp. 899-908, 2003

[5] Güler NF, Übeyli ED, Güler.'Recurrent neural networksemploying Lyapunov exponents for EEG signal classification",Expert Syst Appl. 2005; 29(3):506-14

[6] Varun Bajaj, Ram Bilas Pachori "Epileptic Seizure Detection Based on the Instantaneous Area of Analytic Intrinsic Mode Functions of EEG Signals," Biomed Eng Lett, vol. 3, pp. 17-21, 2013

[7] EEG time timeseries (epilepticdata)(2005,Nov.) [Online], http://www.meb.unibonn.de/epileptologie/science/physik/eegdata.html

[8] Hedi Khammari , Ashraf Anwar, "A Spectral Based Forecasting Tool of Epileptic Seizures " IJCSI International.Journal of Computer Science Issues, Vol. 9, Issue 3, No 3, May 2012

[9] Rami J Oweis and Enas W Abdulhay., "Seizure classification in EEG signals utilizing Hilbert- Huang transform" BioMedical Engineering OnLine 2011, 10:38

[10] lajos losonczi, lászló bakó, sándor-tihamér ,Brassai and lászló-ferenc Márton., "Hilbert-huang transform used for eeg signal analysis ," The 6th edition of the Interdisciplinarity in Engineering International Conference, "Petru Maior" University of Tîrgu Mure, Romania, 2012 


\section{AUTHORS}

Shaik.Jakeer Husain received the B.E degree in Electronics and Communication Engineering from Andhra University, Visakhapatnam in 1996, M.E in Digital System from Osmania University, Hyderabad in 2008 and he is pursuing Ph.D in Digital Signal Processing at JNTU, Hyderabad.He is currently an Associate Professor in Department of Electronics and Communication Engineering at Vidya Jyothi Institute ofTechnology,

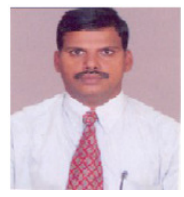
Hyderabad. His research interests include Biomedical Signal Processing and Digital Signal Processing

Dr. K. S. Rao obtained his B. Tech, M. Tech and Ph.D. in Electronics and Instrumentation Engineering in the years 1986, 89 and 97 from KITS, REC Warangal and VRCE Nagpur respectively. He had 25 years of teaching and research experience and worked in all academic positions, presently he is the Director, Anurag Group of Institutions (Autonomous) Hyderabad. His fields of interests are Signal Processing, Neural Networks and VLSI system design.

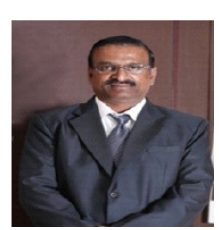

